\title{
Effects of compartmental model structure and long-term inflow pollutograph on model predictions
}

\author{
S. Xu1, A. Wörman² and B. Dverstorp1 \\ ${ }^{1}$ Swedish Radiation Protection Authority (SSI), 17116 Stockholm, Sweden \\ ${ }^{2}$ Swedish University of Agricultural Sciences, Box 7013, 75007 Uppsala, Sweden
}

\begin{abstract}
Model predictions of solute transport in a wetland were compared with tracer experimental data to illustrate that the number of compartments in a compartmental model should be selected according to certain rules to accurately describe the transport process. If the input pulse is short, the model structure affects the output significantly. The temporal moments of the residence time distribution was obtained from a general solution of the compartmental model in the Laplace domain derived with an arbitrary inflow pollutograph. The variance of the residence time can be used as a useful tool to analyse the effect of model structure and long-term inflow pollutograph on the response of the model predictions.
\end{abstract}

\section{INTRODUCTION}

The compartmental system approach is widely used in ecological modelling to quantify flow of energy and materials in ecosystem "compartments" [1]. The fundamental assumption behind compartmental models is instantaneous mixing of solute in the compartment. Often one compartment is used to represent a large ecosystem in which sub-compartments are used to describe ecological objects such as populations, communities and etc. depending on the complexity of the system. Spatial distribution of solutes in the system cannot be represented by using such an approach. However, transport of solutes in the environment by water flow leads to a temporal and spatial distribution of solutes in the system.

Kirchner [2] studied the applicability of compartmental models for simulating the transport of radionuclides in soil and concluded that the number of compartments into which a soil is divided cannot be chosen arbitrarily, but should be set to a specific value defined by the physics of the transport process of water and solutes in the soil. In this study we try to analyse effects of the number of compartments chosen to represent solute transport processes on ecological modelling in different media.

For the simplicity we will not include other processes than those related to solute transport by water flow. Two questions are discussed. One is under what conditions the number of compartments used in the compartmental model, i.e. the structure of the compartmental model, affects the model predictions. The other issue concerns what type of effects may occur in the behaviour of model predictions. The current study attempts to provide a simple approach to examine the effect of model structure and inflow pollutograph on the response of transport processes in a system. This study makes use of the compartmental model, in which the forward transfer rate includes convective transport process.

Model predictions are compared with experimental data obtained from a tracer experiment performed at Ekeby constructed wetland in Sweden to explore the impact of the compartmental model structure on the representation of solute transport. This study differs from previous analyses ([2] and [3]), 
in which the compartmental model is solved for an instantaneous Dirac pulse input, in that the solution accounts for an arbitrary solute input at the upper boundary. In this way, we can analyse the effect of both model structure and long-term inflow pollutograph on the response of solute transport.

\section{THEORY}

\subsection{Model description}

The compartmental forward model for transport of radionuclides in a system that is divided into a series of $N$ compartments is schematically shown in Fig. 1 . With mass balance the dynamics of the system can be described mathematically by the following set of equations:

$$
\begin{gathered}
\frac{d c_{1}(t)}{d t}=-q_{1} c_{1}(t) \\
\frac{d c_{i}(t)}{d t}=-q_{i} c_{i}(t)+q_{i-1} c_{i-1}(t) \quad i=2, \ldots, N
\end{gathered}
$$

where $c_{i}$ is the concentration of solute in compartment $i, t$ is time, $q_{i}$ is the transport rate coefficient from compartment $i$ to compartment $i+1$ and also can be expressed as $q_{i}=1 / \bar{t}_{i}$ in which $\bar{t}_{i}$ is solute residence time in compartment $i, N$ is the $N$ th compartment at outlet.

Here, the initial and boundary condition are given as:

$$
\begin{aligned}
& c(i=1, t)=A_{0}(t) \\
& c(i \geq 1, t=0)=0
\end{aligned}
$$

where $A_{0}$ is a function of an arbitrary inflow pollutograph.

\subsection{Temporal moments of the compartmental model}

The Laplace transform $L(s)$ of a function $c(t)$ is defined as:

$$
\bar{c}=L[c(t)]=\int_{0}^{+\infty} c(t) \cdot e^{-s \cdot t} d t
$$

where $s$ is the Laplace parameter. We may readily verify that the temporal moments can be expressed as

$$
m_{j}=\int_{0}^{\infty} t^{j} c(t) d t=\left.(-1)^{j} \frac{\partial^{j} L[c(t)]}{\partial s^{j}}\right|_{s=0}
$$

Thus temporal moments of order $i, m_{i}$, can be used to formulate the central temporal moments, which express statistical properties of concentration-time-distribution. The zeros moment, $m_{0}$, is the total mass of inflow. The expected residence time, $\bar{t}$, is expressed as:

$$
\bar{t}=m_{1} / m_{0}
$$

The variance of residence time is given as:

$$
\sigma_{t}^{2}=\int_{0}^{+\infty} f(t)(t-\bar{t})^{2} d t=\frac{m_{2}}{m_{0}}-\left(\frac{m_{1}}{m_{0}}\right)^{2}
$$


where $f(t)=\frac{c(t)}{\int_{-\infty}^{+\infty} c(\tau) d \tau}$ in which $\tau$ is the integrand.

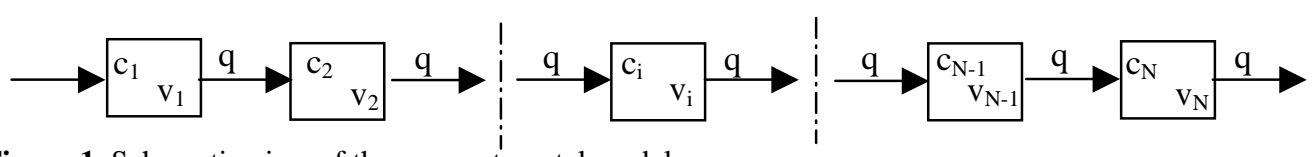

Figure 1. Schematic view of the compartmental model.

If we assume that each compartment has equal volume and $q_{i}$ s are equal. The solution of (1) at $N$ th compartment with initial and boundary conditions (2) and (3) in the Laplace domain is:

$$
\bar{c}_{N}=a_{0}(s) \frac{q^{N-1}}{(s+q)^{N}}
$$

where $a_{0}(s)$ is the Laplace transform of $A_{0}$.

Temporal moments of the residence time distribution associated with the compartmental models can be obtained by application of (5) to (8). By repeated application of (5) to (8) combined with (6) and (7) involves a series of tedious but also relatively simple operations, which leads to the following expressions for the central temporal moments for the compartmental model:

$$
\begin{gathered}
\bar{t}=\bar{t}(0)+\frac{N}{q}=\bar{t}(0)+\sum_{i=1}^{N} \bar{t}_{i} \\
\sigma_{t}^{2}=\sigma_{t}^{2}(0)+\frac{N}{q^{2}}=\sigma_{t}^{2}(0)+\sum_{i=1}^{N} \bar{t}_{i}^{2}
\end{gathered}
$$

where $\bar{t}(0)$ is expected residence time of inflow pollutograph and $\sigma_{t}^{2}(0)$ is variance of residence time of the inflow pollutograph.

\section{MODEL APPLICATION AND DISCUSSION}

\subsection{Effect of model structure}

In this chapter we are going to exemplify how the selected number of compartments affect the response from a solute input to the system. We also indicate the effect of the upper boundary conditions (inflow pollutograph) and model structure on the variance of residence time.

The example makes use of the compartmental model and data obtained from a tracer experiment performed at Ekeby constructed wetland in Eskilstuna County in Sweden. In the experiment 74 GBq of tritium, ${ }^{3} \mathrm{H}_{2} \mathrm{O}$, was injected in one of eight ponds at Ekeby wetland. Fig. 2 shows the measured concentration-time-distribution and computed concentration-time-distributions with different compartments used in the simulation.

The equation system (1) was solved numerically by using the implicit Trapezoidal method [4] with a pulse input. The transport rate coefficient $q$ was determined by using $q=N / 3.5$ where $N$ is the number of compartments and 3.5 is calibrated residence time. Since the experiment duration was in the order of days, the radionuclide decay was neglected in the model description (the half life time for tritium is 12 years). As can been seen from Fig. 2 the model prediction with one compartment deviates significantly from the measured data. When the compartments were set to be four the simulated concentration-time-distributions fits the measured data relatively well. 
One of the findings from the above case study is that to model a transport process using compartmental model the number of compartments cannot be chosen arbitrarily. The number of compartments may be determined by comparing model predictions with tracer test performed at the modelling object. In particular, one of the recommendations of Kadlec and Knight [5] is to use the number of compartments as a fitting parameter in the characterisation of a particular wetland. The tracer experiment can be very costly. An alternative is using complex physical based models (2D or 3D) to study the behaviour of solute transport in an ecological environment of interest.

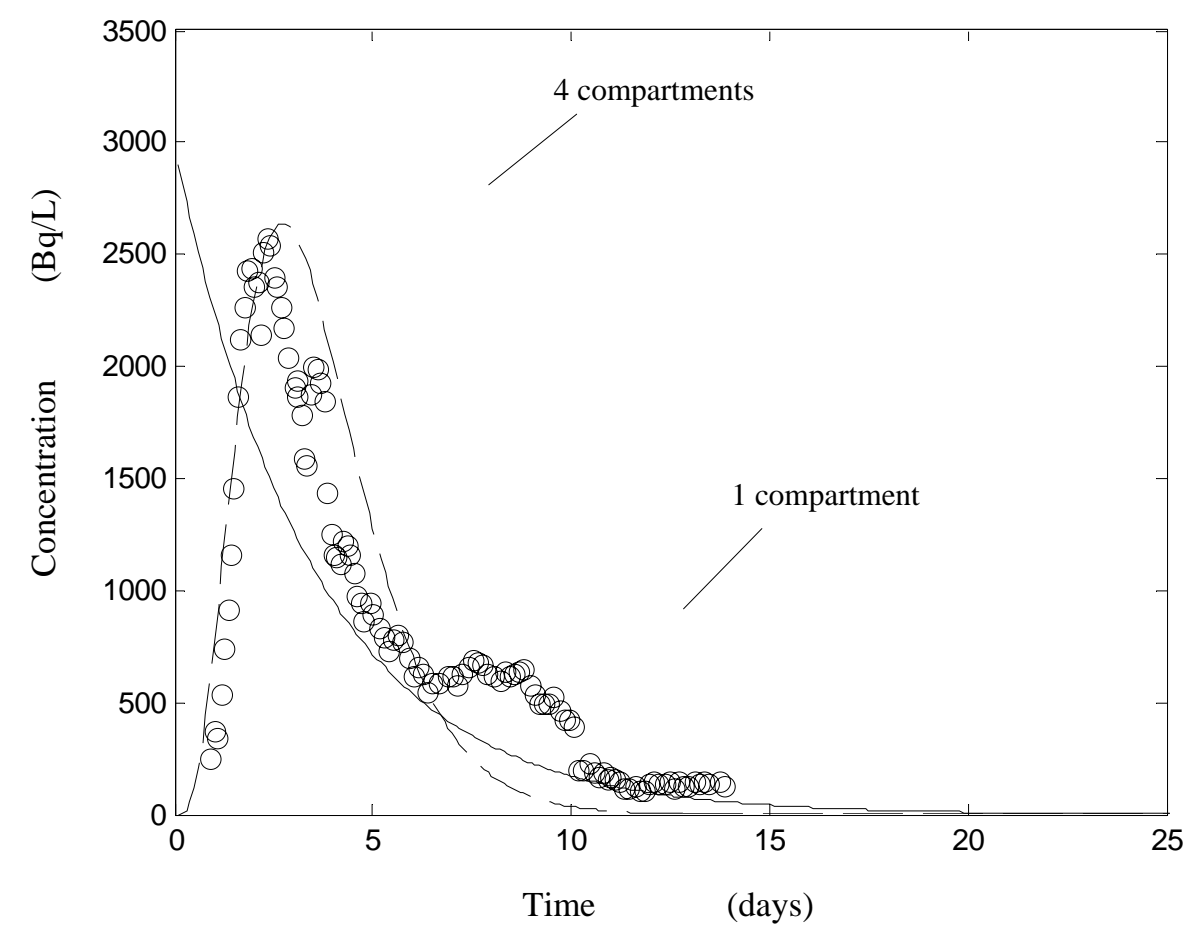

Figure 2. Concentration-time-distribution for tritium from Ekeby wetland experiment: observed data (markers) and predicted curves using the compartmental model with different compartments.

On the other hand one must be aware of the complexity of the wetland hydrology resulting in a situation in which water parcels of incoming water take different routes and time to pass through the wetland due to short cut, hyporheic exchange etc. The compartmental forward model is only a rough approximation of the transport process. This can be seen from Fig. 2 that the second peak of the measured concentration-time-distribution cannot be modelled by the compartmental model.

\subsection{Effect of model structure and long-term inflow}

The example given above is based on a relative short pulse at the upper boundary. However, one may argue that the effect of model structure will decease with duration of the inflow pollutograph. The effect of model structure and inflow pollutograph on the behaviour of the system can be analysed by means of (10). As can be seen from (10) the variance of residence time consists of two parts, one is from the temporal moments of inflow pollutograph and the other from the temporal moments of the 
output of a unit pulse from the compartmental model. If the temporal moments related to the unit pulse through the compartmental model dominate this means that there could be a notable an effect of model structure on the system response. A simple assumption made here is that residence time of solute transport in each compartment is proportional to the retardation factor. Equation (10) can thus be rewritten for a reactive solute as:

$$
\sigma_{t}^{2}=\sigma_{t}^{2}(0)+R^{2} \frac{N}{q^{2}}=\sigma_{t}^{2}(0)+\sum_{i=1}^{N} R_{i}^{2} \bar{t}_{i}^{2}
$$

where $R$ is retardation factor defined in Table 1 for various transport processes and $\bar{t}_{i}$ is the expected residence time for an inert solute in compartment $i$.

Assuming that the inflow pollutograph is a step injection type, the variance of inflow pollutograph can be expressed as:

$$
\sigma_{t}^{2}(0)=\frac{\Delta^{2}}{12}
$$

where $\Delta$ is the duration of the inflow. From (11) and (12) it is clear that the influence of the model structure on the outflow pollutograph decreases as the $\Delta$ tends towards infinity. The example below illustrates how the effect of the model structure depends on $\Delta$.

Consider a case of transport of a medium-sorbing radionuclide, like Caesium, in an aquatic system with a residence time of 14 days for inert water parcels. An estimation of the partition coefficient, $\mathrm{k}_{\mathrm{d}}$, for dissolved Cs in freshwater sediments is $1 \mathrm{~m}^{3} \mathrm{~kg}^{-1}$, the solid density of sediments, $\rho_{\mathrm{s}}$, is taken as $2,600 \mathrm{~kg} / \mathrm{m}^{3}$ and porosity of the sediments, $\varepsilon$, is 0.7 [6]. The factor $\xi V_{z} P T / 2 A$ is taken as 0.3 [10]. This means that the retardation factor for Caesium in wetland is $1.1 \times 10^{3}$. Fig. 3 shows the variance of residence time of the compartmental backflow model as a function of duration of inflow. As can be seen if the duration of the inflow pollutograph is shorter than $\sim 10^{2}$ years, the number of compartments has a significant effect on the variance of the residence time.

Table 1. Retardation coefficients for different media.

\begin{tabular}{|c|c|c|c|}
\hline Medium & Retardation factor, $\boldsymbol{R}$ & Definition & References \\
\hline Bedrock & $1+2 Z \frac{\varepsilon}{h}\left(1+\frac{\rho}{\varepsilon} k_{d}\right)$ & $k_{d}=\left.\left(c_{w} / c_{m}\right)\right|_{e}$ & [7] \\
\hline $\begin{array}{c}\text { Soil and lake } \\
\text { sediments }\end{array}$ & $\left(1+K_{D}\right)$ & $K_{D}=\left(c_{p} / c_{d}\right)_{e}$ & [8] and [9] \\
\hline Streams and rivers & $1+\left(1+K_{B}\right) \frac{\xi V_{z} P T}{2 A}$ & $K_{B}=\frac{\rho}{\varepsilon} k_{d}$ & [10] \\
\hline
\end{tabular}

where $Z=$ matrix "depth" in bedrock, $\varepsilon=$ porosity, $h=$ fracture aperture in bedrock, $\rho=$ density, $\xi=$ area reduction factor for the water exchange between a stream and the hyporheic zone, $P=$ wetted parameter of a stream, $V_{z}=$ exchange velocity for water between a stream and the hyporheic zone, $T=$ residence time of inert solutes (water) in the hyporheic zone, $A=$ cross-sectional area of stream, $c_{w}=$ sorbed solute mass per unit solid mass, $c_{m}=$ dissolved mass per unit volume of water, $c_{p}=$ particulate solute mass per unit bulk volume, $c_{d}=$ dissolved solute mass per unit bulk volume and $\left.\right|_{\mathrm{e}}$ denotes 'evaluated at equilibrium'. 


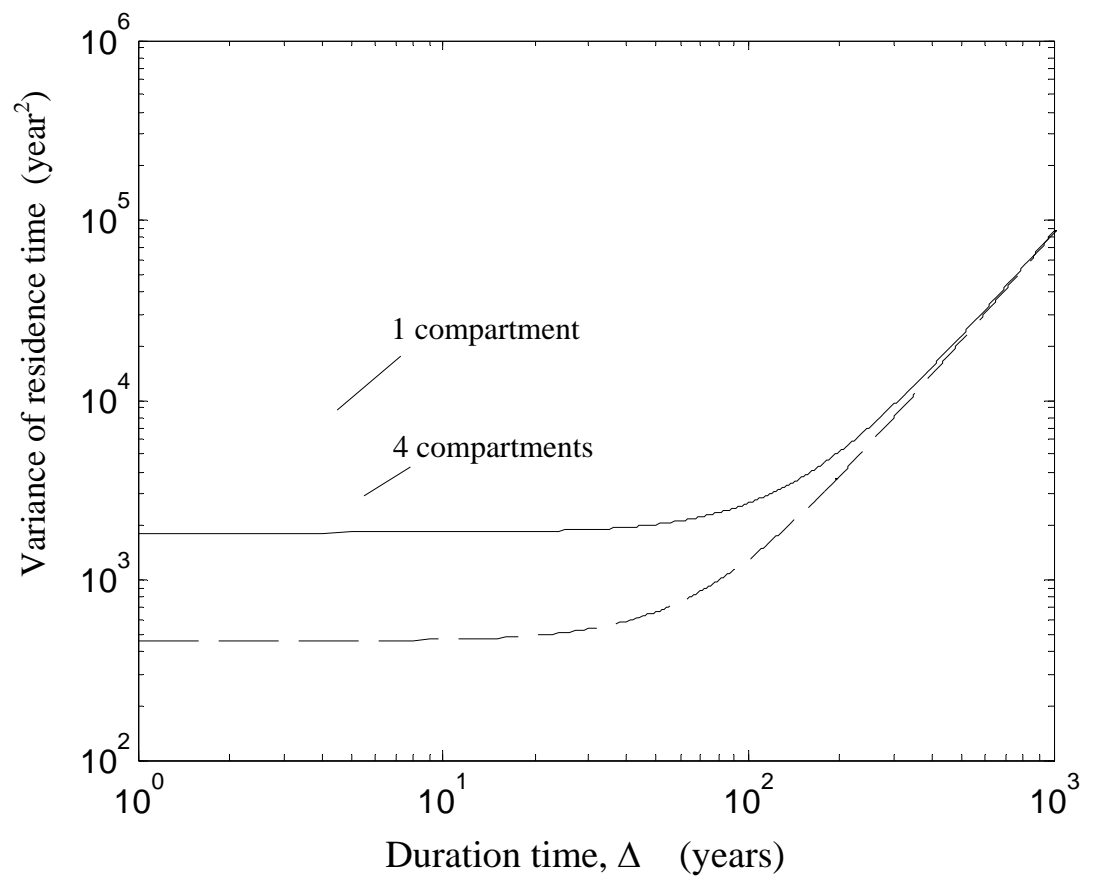

Figure 3. Variance of residence time versus inflow duration.

\section{CONCLUSION}

Significant errors can arise in a compartment model prediction of a solute transport process. Important factors are the number of compartments and duration of the inflow pollutograph. In a given example the predicted concentration-time-distribution deviated significantly from the measured data when only one compartments was chosen.

The effect of the model structure on the outflow pollutograph decreases as the duration of the inflow pollutograph increases. In the example, the effect of model structure was significant for a duration of the inflow pollutograph less than $\sim 10^{2}$ years. The influence of the number of compartments on the response of the model prediction depends also on the retardation factor, i.e. on geochemical factors such as type of radionuclide.

The equation (11) derived in this study can be used as a tool to examine the effect of the model structure and long-term inflow to the system on the variance of residence time distribution. Since we only considered a linear sorption retardation process in this study, a thorough analyses should be performed when one uses simplified compartmental models to predict the behaviour of radionuclides in an ecological environment for radiological assessment purpose.

\section{Acknowledgments}

We would like to thank Håkan Johansson and Anna Lindahl for providing the tracer experimental data. 


\section{References}

[1] Odum, E. P. Fundamentals of ecology. (1971). $3^{\text {rd }}$ ed. W. B. Saunders Co., Philadelphia. 574 pp.

[2] Kirchner, G. (1998). Applicability of compartmental models for simulating the transport of radionuclides in soil. J. Environ. Radioactivity, 38(3), 339-352.

[3] Roemer, M. H. and Durbin, L. D. (1967). Transient response and moments analysis of back-flow cell model for flow systems with longitudinal mixing. Industrial and Engineering Chemistry, Fundamentals 6, 120-129.

[4] Berner, R.A. (1980). Early Diagenesis. Princeton Univ. Press, Princeton, N.J., New Jersey, USA.

[5] Kadlec, R. H. and Knight, R. L. (1996). Treatment wetlands. Lewis Publishers, Boca Raton, New York.

[6] Karlsson, S., Bergström, U. Meili, M. (2001). Models for dose assessments - models adapted to SFR-area, Sweden. TR-01-04, Swedish Nuclear Fuel and Waste Management Co., Stockholm.

[7] Xu, S. and Wörman, A. (1999). Implications of Sorption Kinetics to Radionuclide Migration in Fractured Rock. Water Resources Research, 35(11), 3429-3440.

[8] Kutilek, M. and Nielsen, D. R. (1994). Soil Hydrology, Catena Verlag, Cremlingen-Destedt, Germany.

[9] Burden, R. L. and Faires, J. D. (1993). Numerical analysis. $5^{\text {th }}$ ed. PWS Publishing Company.

[10] Wörman, A., Packman, A., Jonsson, K., Johansson, H. (2002). Effect of Flow-Induced Exchange in Hyporheic Zones on Longitudinal Transport of Solutes in Streams and Rivers. Water Resources Research, 38(1), 2:1-15. 